\title{
On the Number of Clusters in Channel Model
}

\author{
Keziban Akkaya, Celal Alp Tunc, Defne Aktas and Ayhan Altintas \\ Department of Electrical and Electronics Engineering, Bilkent University \\ Bilkent, Ankara, Turkey, TR-06800 \\ Email: keziban@ee.bilkent.edu.tr
}

\begin{abstract}
Typically, scatterers in an environment are not distributed uniformly but rather observed to occur in clusters. Identification of clusters is an issue under discussion. To this end, we study the effect of number of clusters on channel model through computer simulations. We focus on a geometric stochastic directional channel model based on COST259. Fixing a scatterer scenario, we calculate root mean square delay and angular spreads when scatterers are grouped into varying numbers of clusters and study how sensitive these parameters are to the number of clusters used in this channel model.
\end{abstract}

\section{INTRODUCTION}

For the improvement of existing mobile radio systems, realistic channel models are required. In this work, we concentrate on a geometric stochastic channel model based on COST259 [1]. In this model the ray paths are coming from the scatterers distributed in the cell. Number and the density of scatterers differ according to the environment. From the experimental data, it is observed that scatterers tend to occur in groups [2]. Therefore, the scatterers are grouped into clusters. Handling the channel by clustering of scatterers enables us to get a more simplified approach and physically appealing picture.

Clusters of scatterers in a propagation environment are typically identified through channel measurements. To estimate the location of clusters throughout the cell, one approach is to use the angle of arrival data from the measurements. The clusters are then specified according to their contribution to the total received power [3]. According to measurement results, cluster locations and parameters such as propagation delays, angles of arrival and departure for the scatterers within each cluster can be modeled using appropriate probability distribution functions. Afterwards, the impulse response of the geometry based stochastic channel model is evaluated using these parameters [4]. COST259 is one of the geometric channel models and its parameters are based on the number and the location of clusters, and the existence of visibility regions [5]. Since the clustering is the basic concept of modeling, the sensitivity of the channel model to how the scatterers are grouped into clusters is important. The effect of clustering on link capacity is examined in [6]. While examining the effect of clustering, the analytical expressions for angle and delay spread are important to characterize the channel [7]. Using angle and delay spreads, clusters are defined within the environment and the effect of far clusters on root-mean-square (rms) angular spread and channel capacity is discussed in [8].

Our aim in this study is to investigate the effect of number of clusters (NoC) used in the channel model on rms angular spread and rms delay spread (RDS). We use a geometric channel model based on COST259 for an outdoor radio environment. Note that in the COST259 model, NoC is a random parameter. However, in this paper, fixing a scatterer scenario, we group the scatterers into varying NoC and compute the rms angular and delay spreads of the corresponding channel model.

\section{Structure of the System}

We consider a circular cell of radius $R_{\text {cell }}$ where base station (BS) is placed at the center of the cell (origin). Mobile station (MS) can be assumed to be located at any point in the cell. We assume a number of scatterers are distributed over the cell area. These scatterers are grouped into clusters. Each scatterer inside the cluster represents a single multi-path component. The geometrical representation is given in Fig. 1.

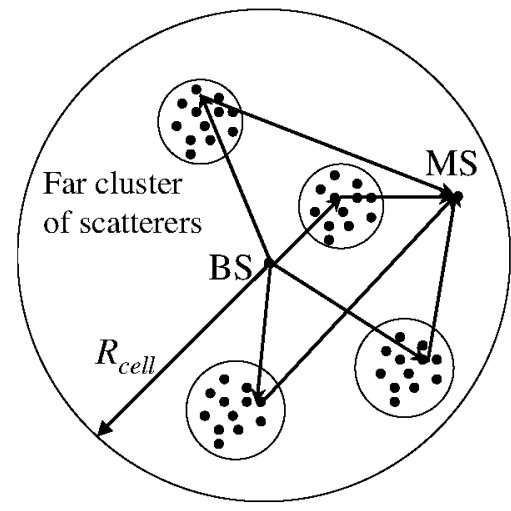

Fig. 1. Geometry based channel model.

The transmitted signal leaves the base station and arrives at the mobile station with a particular delay which depends on the distance signal travels. The delay is calculated for each scatterer inside each cluster. First, we find the distances from the cluster center to the base station and mobile station, separately. The total path length is the sum of these distances $\left(d_{n}\right)$. Then, we find the corresponding delay $\left(\tau_{n}\right)$ for this path as

$$
\tau_{n}=\frac{d_{n}}{c}
$$

where $c$ is the speed of light. The propagation delay associated with the $k$ th scatterer in $n$th cluster is given as

$$
\tau_{n, k}=\tau_{n}+\tau_{a d d, n, k}
$$


where $\tau_{a d d, n, k}$ is the additional delay for the corresponding scatterer. The additional delay for each scatterer inside the cluster is modeled as a Laplacian distributed random variable with probability density function (pdf) [4]:

$$
f\left(\tau_{a d d, n, k}\right)=\frac{1}{\sqrt{2}} \exp \left(-\sqrt{2}\left|\tau_{a d d, n, k}\right|\right)
$$

The rms delay spread is then computed as [9]:

$$
\sigma_{\tau}=\sqrt{\left|\frac{\sum_{n, k} \tau_{n, k}^{2}}{N_{s t}}-\left(\frac{\sum_{n, k} \tau_{n, k}}{N_{s t}}\right)^{2}\right|}
$$

where $N_{s t}$ is the total number of scatterers.

Since COST259 is a directional channel model, we need to compute the angle of each multipath component. Firstly, the angle between the center of the cluster and the mobile station is calculated and denoted by $\bar{\varphi}$. Then, the angle of arrival corresponding to the $k$ th scatterer in $n$th cluster is found from [4] as

$$
\varphi_{n, k}=\bar{\varphi}+\varphi_{a d d, n, k}
$$

where $\varphi_{\text {add }, n, k}$ is the additional shift in angle of arrival for the $k$ th scatterer. This angle is also assumed to have a Laplacian distribution. The rms angular spread is computed in a similar fashion as the rms delay spread.

The impulse response of the channel is then obtained as:

$$
H(t, \varphi)=\sum_{n=1}^{N_{c}} \sum_{k=1}^{N_{s}}\left[e^{j \theta_{n, k}} \delta\left(t-\tau_{n, k}\right) \delta\left(\varphi-\varphi_{n, k}\right)\right]
$$

where $N_{c}$ denotes the number of clusters and $N_{s}$ denotes the number of the scatterers within the cluster and $\theta_{n, k}$ is the phase shift associated with the $\{n, k\}$ th scatterer and is modeled as uniformly distributed.

\section{THE METHOD FOR CLUSTERING OF THE SCATTERERS}

Typically the scatterers are not distributed uniformly within the cell area. However, it is usually not clear how to group the scatterers into clusters for a given scenario.

We use k-means algorithm to group a given set of scatterers into $N_{c}$ clusters [10]. In the k-means algorithm grouping of scatterers into $N_{c}$ clusters is based on the relative Euclidean distances of geometric centroids of clusters to each other. In a recent paper, k-means algorithm with a different distance metric is used to identify clusters from measurement data [11].

In order to study the effect of NoC used in the channel model on channel statistics we vary the number of clusters $N_{c}$, from 2 to $N_{s t}$, the number of total scatterers, and compute the RDS and rms angular spread for each value of $N_{c}$. Note that $N_{c}=N_{s t}$ corresponds to the case where each scatterer is a cluster itself.

\section{NUMERICAL RESULTS}

The scatterer scenario considered is illustrated in Fig. 2 (a). Radius of the cell (RC) is taken as $2 \mathrm{~km}$. Two different scenarios are considered where the mobile station is located at $(0,-500)$ and $(0,-1500)$, so that the mobile distance from the base station (MD) is $0.5 \mathrm{~km}$ and $1.5 \mathrm{~km}$, respectively. Figs. 3 and 4 compare the RDS as a function of NoC for two different mobile locations and two values of total number of scatterers (NofSc). For the computation of rms values the results are averaged over 2,000 realizations.

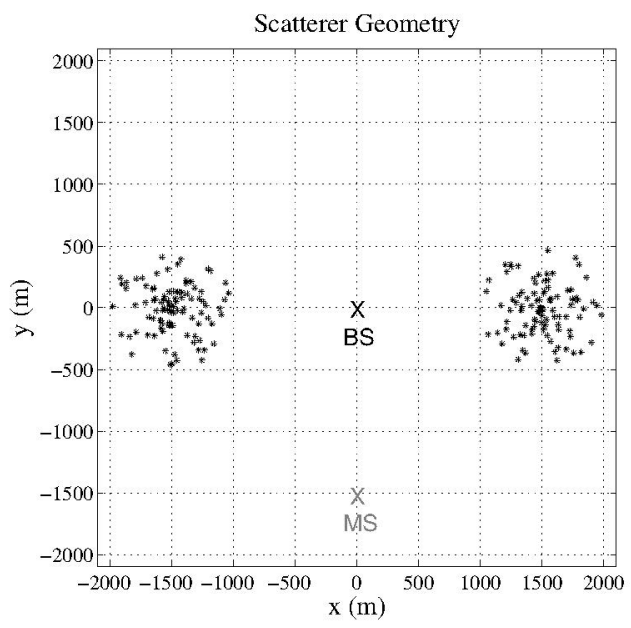

(a) The scenario where 200 scatterers are randomly distributed throughout the cell.

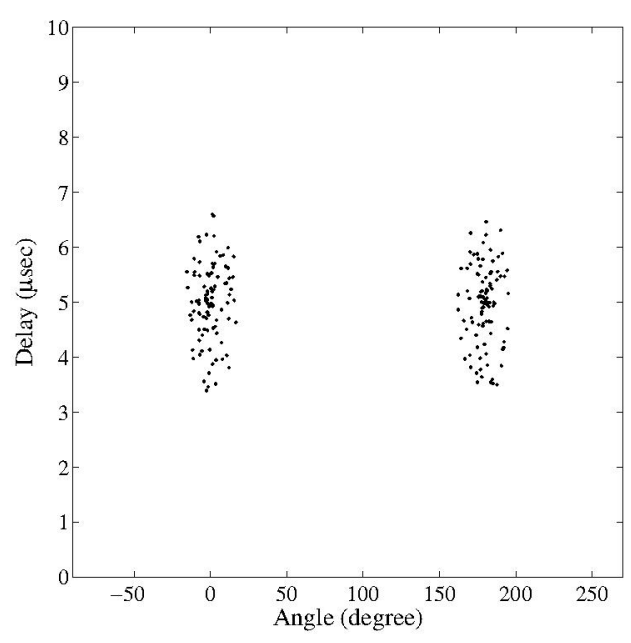

(b) Delay vs angle distribution for the scenario considered

Fig. 2. Scatterer scenario.

From Figs. 3 and 4, it is observed that as NoC increases, the RDS quickly converges to the limiting value. The limiting value corresponds to the case where each individual scatterer is considered a cluster, i.e., $N_{c}=N_{s t}$. It is noted that the limiting value depends on the number of scatterers (NofSc), as shown in Figs. 3 and 4, since the scatterer locations are different in the two scenarios. However, when NoC is small (e.g. less than 20), the RDS is more dependent on NoC, since path delays of scatterers inside a cluster do not approximate the actual delay values close enough. It should be noted that most geometric channel models such as COST259 assumes small NoC.

Results for the rms angular spread are slightly different from RDS. Observing Figs. 5 and 6, it is seen that the rms angular 


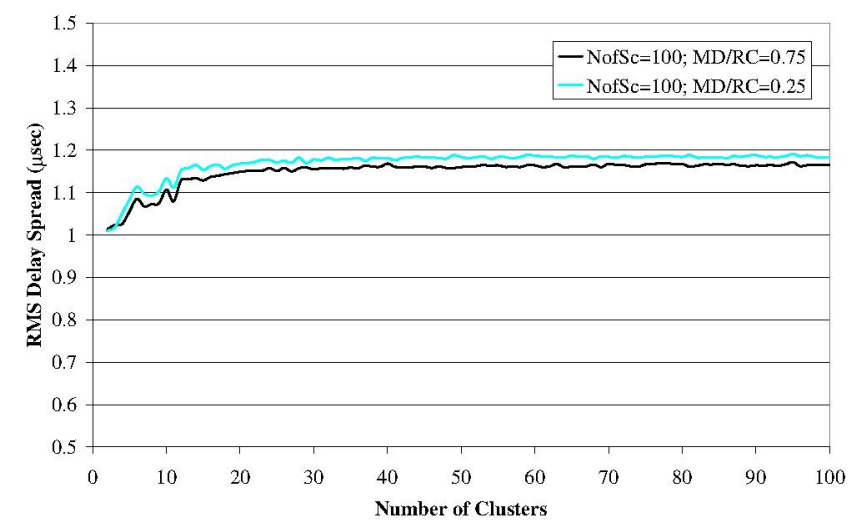

Fig. 3. RDS for total number of scatterers is equal to 100 .

spread varies notably around the limiting value as a function of NoC. This variation is more pronounced for low NoC values. So the channel is more dependent on clustering compared to the RDS results. This may be due to the fact that in k-means algorithm, clusters are selected based on Euclidean distance metric only.

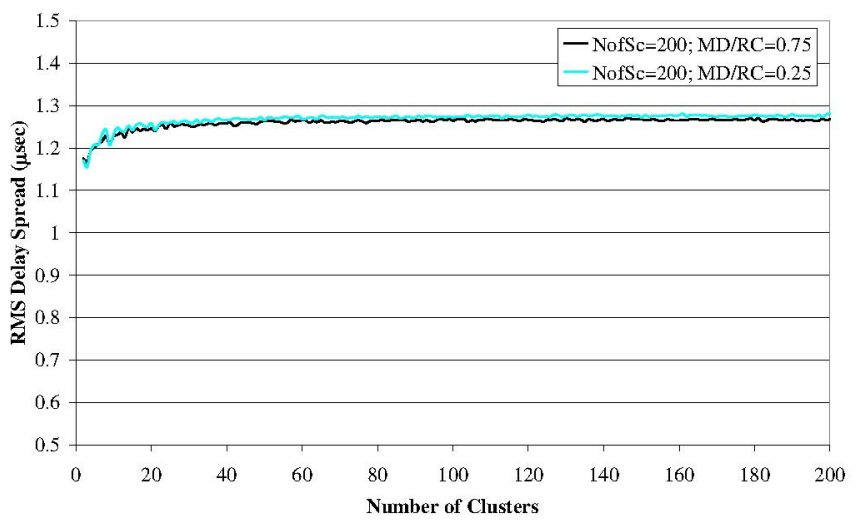

Fig. 4. RDS for total number of scatterers is equal to 200 .

\section{CONCLUSION}

Clustering of scatterers is a useful tool for simulating mobile radio channels. However, identification of clusters from the measurement data is an open problem. In this paper, we study how sensitive channel parameters are to the number of clusters used in a geometric stochastic channel model. Fixing a scatterer scenario, we vary the number of clusters that the scatterers are distributed into and calculate the rms delay spread and rms angle of arrival spread of a geometric channel model based on COST259. Simulation results demonstrate that while the rms delay spread increases as the number of clusters increases, it converges to the limiting value quickly. However,

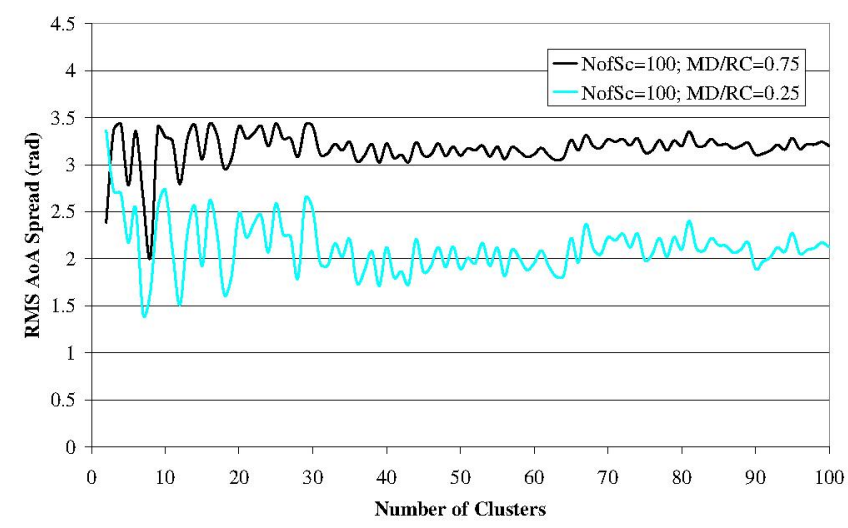

Fig. 5. Rms angle of arrival spread for total number of scatterers is equal to 100 .

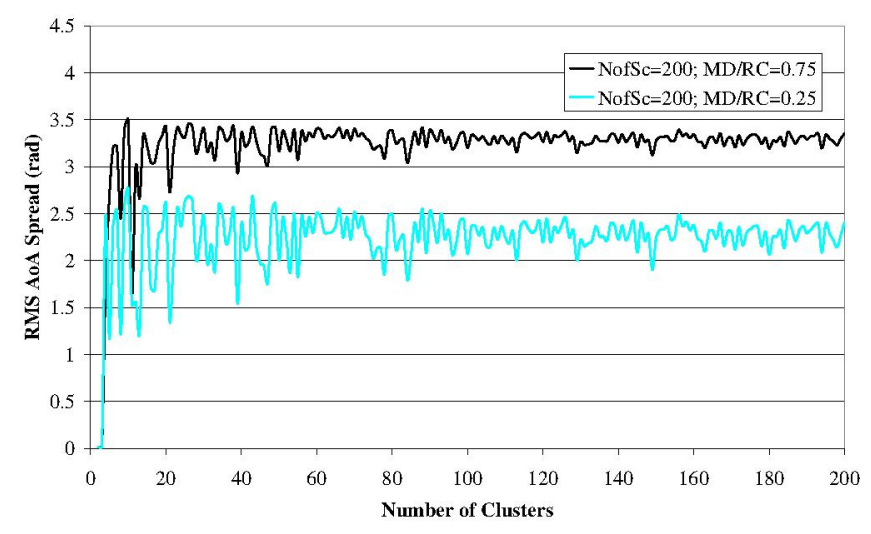

Fig. 6. Rms angle of arrival spread for total number of scatterers is equal to 200 .

the rms angular spread is more sensitive to the number of clusters used in the channel model.

\section{ACKNOWLEDGMENT}

This work has been conducted within NEWCOM Network of Excellence in Wireless Communications funded through the European Community (EC) 6th Framework Program.

\section{REFERENCES}

[1] L.M. Correia, Wireless Flexible Personalised Communications, John Wiley\&Sons, Mar. 2001.

[2] J. Fuhl, A.F. Molisch, and E. Bonek, 'Unified channel model for mobile radio systems with smart antennas", Radar, Sonar and Navigation, IEE Proceedings, vol. 145 , no. 1 , pp.32, Feb. 1993.

[3] L. Vuokko, P. Vainikainen, and J. Takada, "Experimental study of clusters in urban macrocellular environments," COST 273 TD (03)176, Prague, Czech Republic, Sept. 2003.

[4] I. Cosovic, and R. Raulefs, "Comparison and implementation of the directional geometric-stochastic based channel model," 10th Telecommunications Fortum TELROR'2002, Belgrade, Yugoslavia, Nov. 2002. 
[5] H. Asplund, A.F. Molisch, M. Steinbauer, and N.B. Mehra, "Clustering of scatterers in mobile radio channels - Evaluation and modeling in the COST259 directional channel model", in Proc. IEEE International Conference on Communications (ICC2002), vol. 2, pp. 901-905, Apr. 2002.

[6] K.H. Li, M.A. Ingram, and A.V. Nguyen, "Impact of clustering in statistical indoor propagation models on link capacity," IEEE Trans. Commun., vol. 50, no. 4, Apr. 2002.

[7] Y. Chen and V.K. Dubey, "A generic channel model in multi-cluster environments," in Proc. IEEE Vehicular Technology Conf., vol. 1, , pp. 217-221, Apr. 2003.

[8] A.F. Molisch, "Effect of far scatterer clusters in MIMO outdoor channel models," in Proc. IEEE Vehicular Technology Conference (VTC), vol. 1, pp. 534-538, April 2003.

[9] T. S. Rappaport, Wireless Communications: Principles and Practice, 2nd ed., Prentice Hall, 2001.

[10] A. Likas, N. Vlassis, and J.J. Verbeek, "The global k-means clustering algorithm," Pattern Recognition, vol. 36, pp. 451 - 461, 2003.

[11] N. Czink, P. Cera, J. Salo, E. Bonek, J.-P. Nuutinen, and J. Ylitalo, "Improving clustering performance using multipath component distance," Electronics Letters, vol. 42, no.1, 5th January 2006. 